\title{
Byzantine Generalized Lattice Agreement
}

\author{
Giuseppe Antonio Di Luna* , Emmanuelle Anceaume ${ }^{\dagger}$, Leonardo Querzoni* \\ *: DIAG, Sapienza University, Roma, Italia. \{diluna, querzoni\}@diag.uniroma1.it \\ $\dagger$ : CNRS, Univ Rennes, Inria, IRISA, France. emmanuelle.anceaume@irisa.fr
}

\begin{abstract}
The paper investigates the Lattice Agreement (LA) problem in asynchronous systems. In LA each process proposes an element $e$ from a predetermined lattice, and has to decide on an element $e^{\prime}$ of the lattice such that $e \leq e^{\prime}$. Moreover, decisions of different processes have to be comparable (no two processes can decide two elements $e$ and $e^{\prime}$ such that $\left(e \not \leq e^{\prime}\right) \wedge\left(e^{\prime} \not \leq e\right)$ ).

It has been shown that Generalized LA (i.e., a version of LA proposing and deciding on sequences of values) can be used to build a Replicated State Machine (RSM) with commutative update operations. The key advantage of LA and Generalized LA is that they can be solved in asynchronous systems prone to crash-failures (this is not the case with standard Consensus).

In this paper we assume Byzantine failures. We propose the Wait Till Safe (WTS) algorithm for LA, and we show that its resilience to $f \leq(n-1) / 3$ Byzantines is optimal. We then generalize WTS obtaining a Generalized LA algorithm, namely GWTS. We use GWTS to build a RSM with commutative updates. Our RSM works in asynchronous systems and tolerates $f \leq(n-1) / 3$ malicious entities. All our algorithms use the minimal assumption of authenticated channels. When the more powerful public signatures are available, we discuss how to improve the message complexity of our results (from quadratic to linear, when $f=\mathcal{O}(1)$ ). At the best of our knowledge this is the first paper proposing a solution for Byzantine LA that works on any possible lattice, and it is the first work proposing a Byzantine tolerant RSM built on it.
\end{abstract}

\section{Introduction}

State machine replication (RSM) is today the foundation of many cloud-based highly-available products: it allows some service to be deployed such to guarantee its correct functioning despite possible faults. In RSM, clients issue operation requests to a set of distributed processes implementing the replicated service, that, in turn, run a protocol to decide the order of execution of incoming operations and provide clients with outputs. Faults can be accidental (e.g. a computer crashing due to a loss of power) or have a malicious intent (e.g. a compromised server). Whichever is the chosen fault model, RSM has proven to be a reliable and effective solution for the deployment of dependable services. RSM is usually built on top of a distributed Consensus primitive that is used by processes to agree on the order of execution of requests concurrently issued by clients. The main problem with this approach is that Consensus is impossible to achieve deterministically in a distributed settings if the system is asynchronous and even just a single process may fail by crashing [1]. This led the research community to study and develop alternative solutions based on the relaxation of some of the constraints, to allow agreement to be reached in partially synchronous systems with faulty processes by trading off consistency with availability.

An alternative approach consists in imposing constraints on the set of operations that can be issued by clients, i.e. imposing updates that commute. In particular, in 2012 Faleiro et al. 2 introduced a RSM approach based on a generalized version of the well known Lattice Agreement (LA) problem, that restricts the set of allowed update operations to commuting ones 3 . They have shown that commuting replicated data types (CRDTs) can be implemented with an RSM approach in asynchronous settings using the monotonic growth of a join semilattice, i.e., a partially ordered set that defines a join (least upper bound) for all element pairs (see Figure 1 for an example). A typical example is the implementation of a dependable counter with add and read operations, where updates (i.e. adds) are commutative.

In the Lattice Agreement problem, introduced by Attiya et al. 4], each process $p_{i}$ has an input value $x_{i}$ drawn from the join semilattice and must decide an output value $y_{i}$, such that (i) $y_{i}$ is the join of $x_{i}$ 
and some set of input values and (ii) all output values are comparable to each other in the lattice, that is form a chain in the lattice (see Figure 1). Lattice Agreement describes situations in which processes need to obtain some knowledge on the global execution of the system, for example a global photography of the system. In particular Attiya et al. [4] have shown that in the asynchronous shared memory computational model, implementing a snapshot object is equivalent to solving the Lattice Agreement problem.

Differently from Consensus, Lattice Agreement can be deterministically solved in an asynchronous setting in presence of crash failures. Faleiro et al. [2] have shown that a majority of correct processes and reliable communication channels are sufficient to solve Lattice Agreement, while Garg et al. [5] proposed a solution that requires $\mathcal{O}(\log n)$ message delays, where $n$ is the number of processes participating to the algorithm. The very recent solution of Skrzypczak et al. [6] considerably improves Faleiro's construction in terms of memory consumption, at the expense of progress.

In the Generalized Lattice Agreement (GLA) problem processes propose an infinite number of input values (drawn from an infinite semilattice) and decide an infinite sequence of output values, such that, all output values are comparable to each other in the lattice i.e. form a chain (as for Lattice Agreement); the sequence of decision values are non-decreasing, and every input values eventually appears in some decision values. Solving GLA in asynchronous distributed systems reveals to be very powerful as it allows to built a linearizable RSM of commutative update operations 2].

Despite recent advancements in this field, to the best of our knowledge no general solution exists that solves Lattice Agreement problems in an asynchronous setting with Byzantine faults. In the present paper we continue the line of research on Lattice Agreement in asynchronous message-passing systems by considering a Byzantine fault model, that is a model where processes may exhibit arbitrary behaviors.

We first introduce the LA specifications that takes into account Byzantine faults. Then we propose an algorithm, namely Wait Till Safe (WTS), which, in presence of less than $(n-1) / 3$ Byzantine processes, guarantees that any correct process decides in no more than $5+2 f$ message delays with a global message complexity in $\mathcal{O}\left(n^{2}\right)$ per process. We show that $(n-1) / 3$ is an upper bound. Our algorithm makes use of a Byzantine reliable broadcast primitive to circumvent adversarial runs where a Byzantine process may induce correct processes to deliver different input values. The algorithm is wait-free, i.e., every process completes its execution of the algorithm within a bounded number of steps, regardless of the execution of other processes. Note that Nowak and Rybicki [7] recently proposed a solution for a more constrained specification of LA in a Byzantine setting where correct processes are not allowed to decide values that contain inputs from Byzantine processes; Similarly to previous contributions in the area of Byzantine fault-tolerant agreement [8], our specification allows that input values proposed by Byzantine processes may be included in some decisions from some correct processes; this choice allows us to sidestep the impossibility results of 8 and makes our solution suitable in important application use cases where the set of input values is infinite (e.g. natural numbers).

We then go a step further by proposing an algorithm, namely Generalized Wait Till Safe (GWTS), to solve GLA in a Byzantine fault model. Here the challenge is twofold: first, we need to guarantee that, despite the fact that input values are proposed in tumbling batches, Byzantine processes cannot keep rejecting all new proposals under the pretext that they are not comparable with the current ones. Second, we must ensure that adversarial processes cannot progress much faster than all the other correct processes (i.e., output decision values faster than correct processes) which would allow them to prevent all correct processing from proposing their own values. Our "wait until safe" strategy guarantees that each correct process performs an infinite sequence of decisions, and for each input received at a correct process, its value is eventually included in a decision. Our algorithm is wait-free and is resilient to $f \leq(n-1) / 3$ Byzantine processes.

We present the construction of a RSM for objects with commuting update operations that guarantees both linearizability and progress in asynchronous environments with Byzantine failures.

Finally, we sketch the main lines of a signature-based version of our algorithms which takes advantage of digital signatures to reduce the message complexity to $\mathcal{O}(n)$ per process, when $f=\mathcal{O}(1)$.

To the best of our knowledge this is the first paper proposing a solution for Byzantine LA that works on any possible lattice, and it is the first work proposing a Byzantine tolerant RSM built on it.

The rest of this paper is organized as follows: Section 2 discussed the related works; Section 4 illustrates the necessity of at least $(3 f+1)$ processes, while Section 3 describes the system model; Section 5 and Section 6 introduce the algorithms for Byzantine LA and Byzantine GLA, respectively; Section 7 describe the construction of a byzantine tolerant RSM; Section 8 sketches a signature-based variant of our solutions and, finally, Section 9 concludes the paper. 


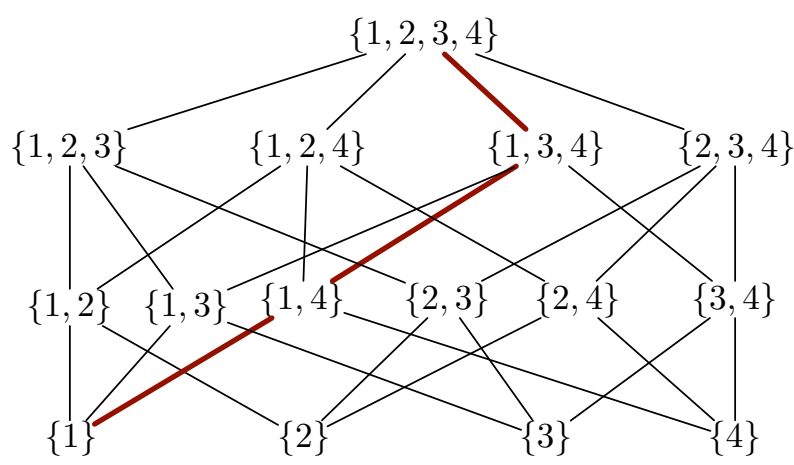

Figure 1: Hasse diagram of the semilattice induced over the power set of $\{1,2,3,4\}$ using the union operation as join. Taken two elements $e, e^{\prime}$ of the lattice if $e<e^{\prime}$, then $e$ appears lower in the diagram than $e^{\prime}$ and there is an "upward" path, going from lower points to upper points, connecting $e$ to $e^{\prime}$ (e.g., $\{1\} \leq\{1,3,4\}$, but $\{2\} \not \leq\{3\})$. Any two elements $e, e^{\prime}$ of the semilattice have a join $e \oplus e^{\prime}=e \cup e^{\prime}$ and $e \oplus e^{\prime} \geq e, e^{\prime}$ (e.g., $\{1\} \oplus\{2,3\}=\{1,2,3\}$ ). The red edges indicate the chain (i.e., sequence of increasing values) selected by the Lattice Agreement protocol.

\section{Related Work}

Lattice Agreement has been introduced by Attiya et al [4] to efficiently implement an atomic snapshot object 9,10 . Their construction is such that each scan or collect operation requires $\mathcal{O}(1)$ execution of LA and uses $\mathcal{O}(n)$ read/write registers. Then Faleiro 2 have shown that GLA is a very interesting abstraction to build RSMs with strong consistency properties, i.e., linearizability of its operations, and liveness guarantees in asynchronous systems. Very recently Nowak and Rybicki 7 have studied LA in presence of Byzantine faults. As previously introduced, their specifications of LA is more restrictive than the one we propose since it does not allow decisions to contain values proposed by Byzantine processes. We argue that our Lattice Agreement specification is more adapted to build RSM on top of the LA algorithm. Removing a value proposed by a Byzantine process might not be desirable: think about an RSM that implements an object shared by different organizations, it could be a breach of contract to selectively avoid certain updates even when the sender misbehaved. A second reason is more technical and it stems from the interaction between the impossibility results introduced by the specifications of 7 and how an RSM is implemented using GLA [2]. Following 2] to implement the RSM we take the power set of all possible updates and we construct a lattice on it using as join the union operation. As an example, let us suppose that we want to build a set counter data type, and let us assume that clients issue four update operations add(1), add(2), add(3), add(4) interleaved with reads. In this case our semilattice is the one in Figure 1, and a Lattice Agreement algorithm will ensure that each read will see values on a single chain, the red one in the figure. Thanks to this, different reads will see "growing" versions of the counter that are consistent snapshots (e.g., if someone reads $\{1\}$, the other could read $\{1,4\}$, but it can not read $\{4\}$ ). Such a semilattice has a breadth ${ }^{1}$ of 4 , actually, each semilattice obtained using as join operation the union over the power set of a set of $k$ different values has breadth $k$. Therefore to satisfy the specifications of [7] using the semilattice in Figure 1 we should have at least 5 processes participating to Lattice Agreement. Unfortunately, it is often the case that the number of possible update operations is larger than the processes running the LA. In the set counter data type, we may have an add $(x)$ for any $x \in \mathbb{N}$, in such setting the specifications of 7 is impossible to implement. Our specifications circumvent such impossibility.

\section{Model and Problem Definition}

We have a set $P:\left\{p_{0}, p_{1}, \ldots, p_{n-1}\right\}$ of processes. They communicate by exchanging messages over asynchronous authenticated reliable point-to-point communication links (messages are never lost on

\footnotetext{
${ }^{1}$ Formally, the breadth of a semilattice $L=(V, \oplus)$ is the largest $n$, such taken any set of $U \subseteq V$ of size $n+1$ we have $\bigoplus U=\bigoplus K$ where $K$ is a proper subset of $U$.
} 
links, but delays are unbounded). The communication graph is complete: there is a communication link between each pair of processes.

We have a set $F \subset P$ of Byzantine processes, with $|F| \leq f$. Byzantine processes deviate arbitrarily from the algorithm. We assume $|P| \geq 3 f+1$ (we show that such assumption is needed in Section 4).

In Section 8, we assume that there exists a public-key infrastructure, and that each process is able to sign a message, in such a way that each other process is able to unambiguously verify such signature. In this case Byzantine processes are not able to forge a valid signature for a process in $C=P \backslash F$ (that is the computational capability of processes in $F$ is polynomially bounded). We remark that signatures are only used in the algorithms presented in Section 8

\subsection{The Byzantine Lattice Agreement Problem}

Each process $p_{i} \in C$ starts with an initial input value pro $_{i} \in E \subseteq V$. Values in $V$ form a join semilattice $L=(V, \oplus)$ for some commutative join operation $\oplus$ : for each $u, v \in V$ we have $u \leq v$ if and only if $v=u \oplus v$. The set $E$ is a set of lattice values that can be proposed by a process. Given $V^{\prime}=\left\{v_{1}, v_{2}, \ldots, v_{k}\right\} \subseteq V$ we have $\oplus V^{\prime}=v_{1} \oplus v_{2} \oplus \ldots \oplus v_{k}$.

The task that processes in $C$ want to solve is the one of Lattice Agreement, and it is formalised by the following properties:

- Liveness: Each process $p_{i} \in C$ eventually outputs a decision value $\operatorname{dec}_{i} \in V$;

- Stability: Each process $p_{i} \in C$ outputs a unique decision value $\operatorname{dec}_{i} \in V$;

- Comparability: Given any two pair $p_{i}, p_{j} \in C$ we have that either $\operatorname{dec}_{i} \leq \operatorname{dec}_{j}$ or $d e c_{j} \leq \operatorname{dec}_{i}$;

- Inclusivity: Given any correct process $p_{i} \in C$ we have that pro $_{i} \leq \operatorname{dec}_{i}$;

- Non-Triviality: Given any correct process $p_{i} \in C$ we have that $\operatorname{dec}_{i} \leq \bigoplus(X \cup B)$, where $X$ is the set of proposed values of all correct processes $\left(X:\left\{\right.\right.$ pro $_{i} \mid$ with $\left.\left.p_{i} \in C\right\}\right)$, and $B \subseteq E$ is $|B| \leq f$.

In the rest of the paper we will assume that $L$ is a semi-lattice over sets ( $V$ is a set of sets) and $\oplus$ is the set union operation. This is not restrictive, it is well known 11 that any join semi-lattice is isomorphic to a semi-lattice of sets with set union as join operation.

\section{Necessity of at least $(3 f+1)$ processes}

We first show that our specification can only be satisfied when there are at least $(3 f+1)$ processes. Interestingly, this bound holds even if we weaken our specification by removing Inclusivity property.

Theorem 1. Let $\mathcal{A}$ be any asynchronous distributed, and Byzantine tolerant algorithm that solves Byzantine Lattice Agreement when $f$ processes are Byzantine. We have that $\mathcal{A}$ needs at least $(3 f+1)$ processes. This holds even if we drop the Inclusivity property from the specification.

Proof. (Sketch) We first show the result for $f=1$. Let $\mathcal{A}$ be a correct algorithm using 3 processes and tolerating a Byzantine process. The processes are $\left\{p_{1}, p_{2}, p_{b i z}\right\}$, and $p_{b i z}$ is the Byzantine. Let us consider a run where we delay the messages between $p_{1}$ and $p_{2}$. Still $\mathcal{A}$ has to make $p_{1}$ and $p_{2}$ decide before they exchange any message: each one of them could not exclude that the other is the Byzantine process. Suppose also that the byzantine pretend to have proposed value $v_{b i z_{1}}$ while talking with $p_{1}$, and $v_{b i z_{2}}$ while talking with $p_{2}$. Therefore $p_{1}$ has to make a decision seeing only its value $v_{1}$ and $v_{b i z_{1}}$. Also $p_{2}$ sees only values $v_{2}$ and $v_{b i z_{2}}$. Now we have the following possibilities:

- Each of them outputs a decision that contains both values, but then decisions are not comparable.

- Each of them outputs a decision that contains only its proposed value, but then the decisions are not comparable.

- Each of them outputs a decision that contains only the Byzantine value, also in this case the decision are not comparable. 
- One of them, let us say $p_{1}$ outputs $v_{1}$, and the other outputs $v_{2}, v_{b i z_{2}}$. It is clear that also this case does not solve the problem.

For $f>1$ we use the standard simulation argument: given an algorithm that solves the problem with $3 f$ processes we could simulate it using only 3 processes (each of the three internally runs $f$ copies).

\section{$5 \quad$ Algorithm Wait Till Safe (WTS)}

The Wait Till Safe algorithm (Algorithms 1 and 2 is divided in two phases: an initial Values Disclosure Phase where processes are asked to declare to the whole system values they intend to propose, and then a Deciding Phase where processes agree on which elements of the lattice can be decided on the basis of the proposed values. For the sake of clarity, processes are divided in proposers that propose an initial value, and then decide one decision value, and acceptors which help proposers decide. This distinction does not need to be enforced during deployment as each process can play both roles at the same time.

The main idea in the Values Disclosure Phase is to make any proposer disclose its proposed value by performing a Byzantine reliable broadcast. The reliable broadcast prevents Byzantine processes from sending different messages to processes 12, 13. The exact specification of this broadcast is in 14]. In the pseudocode the broadcast primitive is represented by the RELIABLEBrodCAST (used for reliably broadcast messages) function and RBCASTDELIVERY event (that indicates the delivery of a message sent with the reliable broadcast).

Values delivered at each process are saved in a $S v S$ (Safe-values Set). A process moves to the next phase as soon as he receives values from at least $(n-f)$ proposers. Waiting for $(n-f)$ messages is not strictly necessary, but allows us to show a bound of $\mathcal{O}(f)$ on the message delays of our algorithm. Note that, from this point on, some operations of Phase 1 could run in parallel with Phase 2 . Thanks to Value Disclosure Phase a process is committed to its value and cannot change its proposal or introduce a new one during the Deciding Phase. During this latter phase, correct processes only handle messages that contain values in $S v S$, i.e. messages for which the $S A F E()$ predicate is true. Messages that do not satisfy this condition are buffered for later use, i.e. if and when all the values they contain will be in $S v S$.

The Deciding Phase is an extension of the algorithm described in 2 with a Byzantine quorum and additional checks used to thwart Byzantine attacks. Each correct proposer $p$ sends its Proposed value to acceptors in a request message (Line 19p). An acceptor receiving a request, sends an ack if the previously Accepted_set is a subset of the value contained in the request, and updates its Accepted_set using the Proposed set in the request (initially, the Accepted_set of an acceptor is the empty set). Otherwise, the acceptor sends a nack containing the Accepted_set, and it updates its Accepted_set with the union of the value contained in $p$ 's request and its Accepted_set. The proposer $p$ decides if it receives $\lfloor(n+f) / 2\rfloor+1$ acks. In case $p$ receives a nack, then $p$ updates Proposed set by taking the union of it and the value contained in the nack. Each time a proposer updates its Proposed_set set it issues a new request.

\subsection{WTS properties}

\subsubsection{Safety properties}

Observation 1. Given any correct process $p_{j}$ its $S v S$ contains at most one value for each process in $P$.

The above observation derives from the specification of reliable broadcast, and the fact that in the disclosure phase each participating process broadcasts a single value. We say that a message $m$ containing a set of proposed values is "safe" for a process $p_{i}$ if such set of values is contained in $S v S$. It is immediate from function at Lines 36 40 that proposers (in state proposing) change their Proposed_set only when they receive safe messages. The analogous holds for the Accepted_set of acceptors.

We say that a value $v$ receives $m$ acks if it is contained in a Proposed_set, that is in turn contained in ack messages in the form $\langle a c k, \cdot, t s\rangle$ sent by $m$ acceptors. The same meaning is intended when we say that Proposed_set receives acks. 

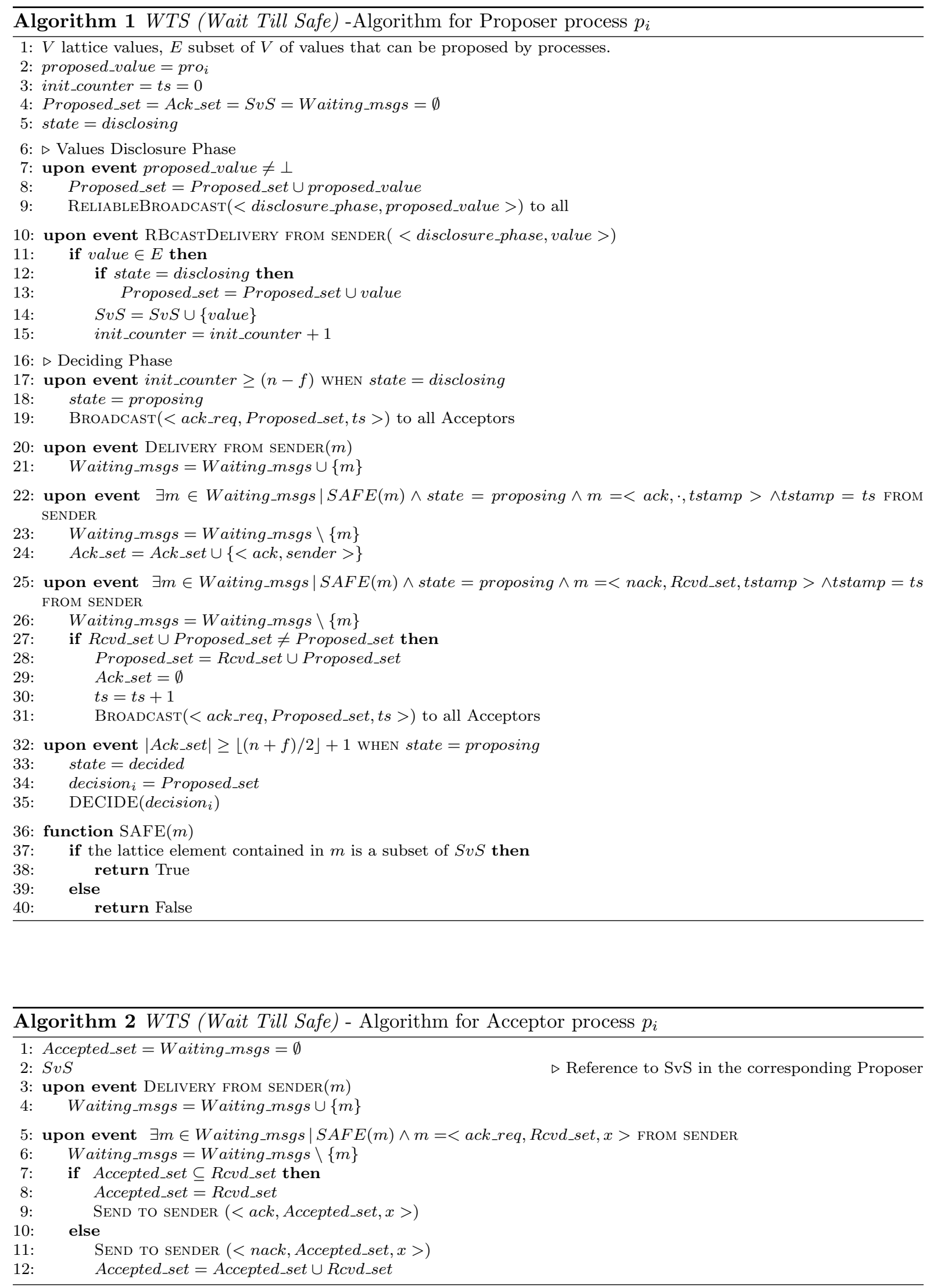
Definition 1. (Commited value) A value $v$ is commited if it received $\lfloor(n+f) / 2\rfloor+1$ acks.

Definition 2. (Commited proposal) A Proposed_set is commited if it received $\lfloor(n+f) / 2\rfloor+1$ acks.

Lemma 1. Let $t$ be the first time at which a value $v$ is commited, we have that any Proposed_set committed after $t$ contains $v$.

Proof. Value $v$ received at least $\lfloor(n-f) / 2\rfloor+1$ acks from acceptors in $C$ (see Alg. 1 Line 39). These acceptors have inserted $v$ in their Accepted_set (see Alg. 2Line 8). Thus by time $t$ a set $Q_{1} \subseteq C$ has $v$ in their Accepted_set. Let Proposed_set be a value committed after $t$, then, by the same above reasoning, we have that Proposed_set received acks from a set of correct acceptors $Q_{2} \subseteq C$, with $\left|Q_{2}\right| \geq\lfloor(n-f) / 2\rfloor+1$. Since $\exists p \in Q_{1} \cap Q_{2}$ (recall that $|C|=(n-f)$ ) we have that Proposed_set contains $v: p$ sent an ack, thus has passed the if at Line 7 of $\mathrm{Alg} 2$

Observation 2. Given any correct process $p_{j}$ its decision ${ }_{j}$ has been committed.

Theorem 2. Let us consider a set of processes, of size at least $(3 f+1)$, executing WTS algorithm. Algorithm WTS enforces: (1) Comparability; (2) Inclusivity; (3) Non-Triviality; (4) Stability.

Proof. We prove each property separately.

1. is implied by Lemma 1 and Observation 2 .

2. derives from the fact that a proposer never removes a value from Proposed_set and from Line 8 .

3. the bound on $B$ derives from the safety of messages and Observation 1 , the fact that $\operatorname{dec}_{i} \leq$ $\bigoplus(X \cup B)$ derives from the fact that a correct process insert in its proposal only values received by messages and its initial proposed value. The fact that $B \subseteq E$ derives from Line 11 of Algorithm 1 .

4. is ensured by Line 33 in proposers.

Note that the Inclusivity and the Comparability imply that, when all correct proposers decide than each value proposed by some correct will be in a decision and that there exists a proposer whose decision includes all values proposed by correct proposers.

\subsubsection{Liveness properties}

Lemma 2. Each message sent by a correct process is eventually safe for any other correct process.

Proof. If a correct process $p_{i}$ sent a message $m$ then the set of values contained in $m$ is a subset of $S v S$ of $p_{i}$. Note that $S v S$ is only updated as result of the reception of a message reliably broadcast in the disclosure phase (Line 14). From the properties of the broadcast eventually each other correct process will obtain a $S v S$ that contains the set of values in $m$, making $m$ safe.

Lemma 3. A correct proposer refines its proposal (executing Line 31) at most $f$ times.

Proof. Each time the proposer executes Line 31 it passes the if at Line 27, thus increasing its proposed set. However, its first proposal, in Line 19, contains at least $|X \cup B|-f$ values. Since there are at most $|X \cup B|$ safe values (from Oservation 1), the claim follows.

Lemma 4. If there is a time $t$ after which a correct proposer $p_{i}$ in state proposing cannot execute Line 31, then $p_{i}$ eventually decides.

Proof. Let $<$ ack_req, Proposed_set, $t s>$ be the last ack request message sent by $p_{i}$. Since $p_{i}$ does not execute Line 31 it means that either $p_{i}$ does not receive any nack, or that any nack $p_{i}$ receives does not allow him to pass the if Line 27. Since $p_{i}$ is correct its message $<a c k \_r e q$, Proposed_set, $t s>$ will reach each correct acceptor. By hypothesis each of them will send a ack, otherwise $p_{i}$ should be able to execute Line 31 (they all handle the ack request by Lemma 2). Once $p_{i}$ receives the acks from the set of correct acceptors will handle them: these messages are safe since the element of the lattice inside each of them is equal to Proposed_set in ack_req and, thus $p_{i}$ decides. 
In the next Theorem (Th. 3) we will show that each correct process eventually commits and decides, we also bound the number of delays needed, by each correct proposer, to reach a decision.

Theorem 3. Let us consider a set of processes, of size at least $(3 f+1)$, executing WTS algorithm. Every correct proposer decides in at most $2 f+5$ message delays.

Proof. The reliable broadcast at Line 9 takes at most 3 message delays. Therefore after three rounds each correct process start its first proposal. Each refinement takes at most 2 message delay, the time needed to broadcast and receive a response. There are $f$ refinements, see Lemma 3 , executed in at most $2 f+2$ message delays, and thus by Lemma 4 after $2 f+5$ message delays a correct decides.

Note that Theorem 3 implies the Liveness property of our Lattice Agreement specification.

\subsubsection{Message complexity}

The Byzantine reliable broadcast used at Line $9 \operatorname{costs} \mathcal{O}\left(n^{2}\right)$ messages $[14$, this cost dominates the other algorithm operations: in the $2 f+5$ delays needed to reach the decision at most $\mathcal{O}(f \cdot n)$ messages are generated.

\section{$6 \quad$ Algorithm Generalized Wait Till Safe (GWTS)}

\subsection{The Generalised Byzantine Lattice Agreement Problem}

In the generalised version of our problem, each process $p_{i}$ receives, asynchronously, input values from an infinite sequence Pro $_{i}=\left\langle\right.$ pro $_{0}$, pro $_{1}$, pro $\left._{2}, \ldots\right\rangle$. Each pro $_{i}$ belongs to a set $E$ of values that can be proposed, note that $E$ is not necessarily finite.

- Liveness: each correct process $p_{i} \in C$ performs an infinite sequence of decisions $D e c_{i}=\left\langle\operatorname{dec}_{0}, \operatorname{dec}_{1}, \operatorname{dec}_{2}, \ldots\right\rangle$;

- Local Stability: For each $p_{i} \in C$ its sequence of decisions is non decreasing (i.e., $\operatorname{dec}_{h} \subseteq d e c_{h+1}$, for any $\left.\operatorname{dec}_{h} \in D e c_{i}\right)$;

- Comparability: Any two decisions of correct processes are comparable, even when they happen on different processes;

- Inclusivity: Given any correct process $p_{i} \in C$, if $\operatorname{Pro}_{i}$ contains a value pro $_{k}$, then pro $_{k}$ is eventually included in $\operatorname{dec}_{h} \in D e c_{i}$;

- Non-Triviality: Given any correct process $p_{i} \in C$ if $p_{i}$ outputs some decision $\operatorname{dec}_{k}$ at time $t$, then $\operatorname{dec}_{k} \leq \bigoplus(\operatorname{Prop}[0: h] \cup B[0: b])$. Where:

$\operatorname{Prop}[0: h]$ is the union of the prefixes, until index $h$, of all sequences Pro $_{i}$ of correct processes; and, $B[0: b]$ is the union of all prefixes, until index $b$, of $f$ infinite sequences $B_{i}$, one for each Byzantine process. Each $B_{i}$ is a sequence of values in $E$.

Intuitively, with Non-Triviality we are bounding the number of values that the Byzantine processes could insert in any decision to a finite number of values.

\subsection{Algorithm Description}

The pseudocode of GWTS is in Algorithms 3.4.

The Generalized Wait Till Safe algorithm is an extension of the WTS algorithm based on the same batching approach proposed in [2]. Input values at proposers are batched until a new decision round starts. Each decision round follows the two-phases approach of WTS. Note that rounds are executed asynchronously at each proposer $2^{2}$

\footnotetext{
${ }^{2}$ The Byzantine reliable broadcast primitive used in 14 is designed to avoid possible confusion of messages in round based algorithms. That is exactly what we need.
} 


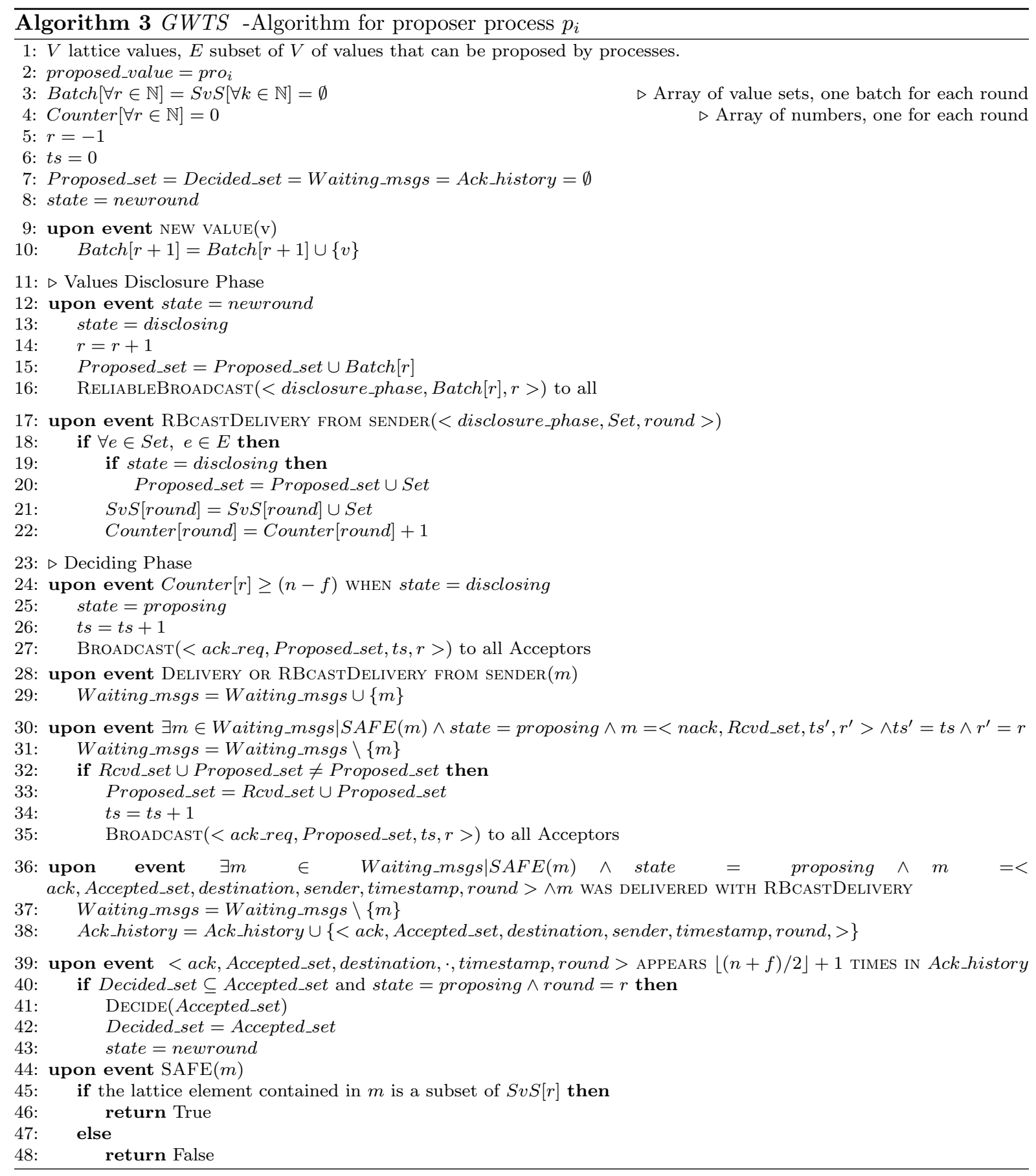




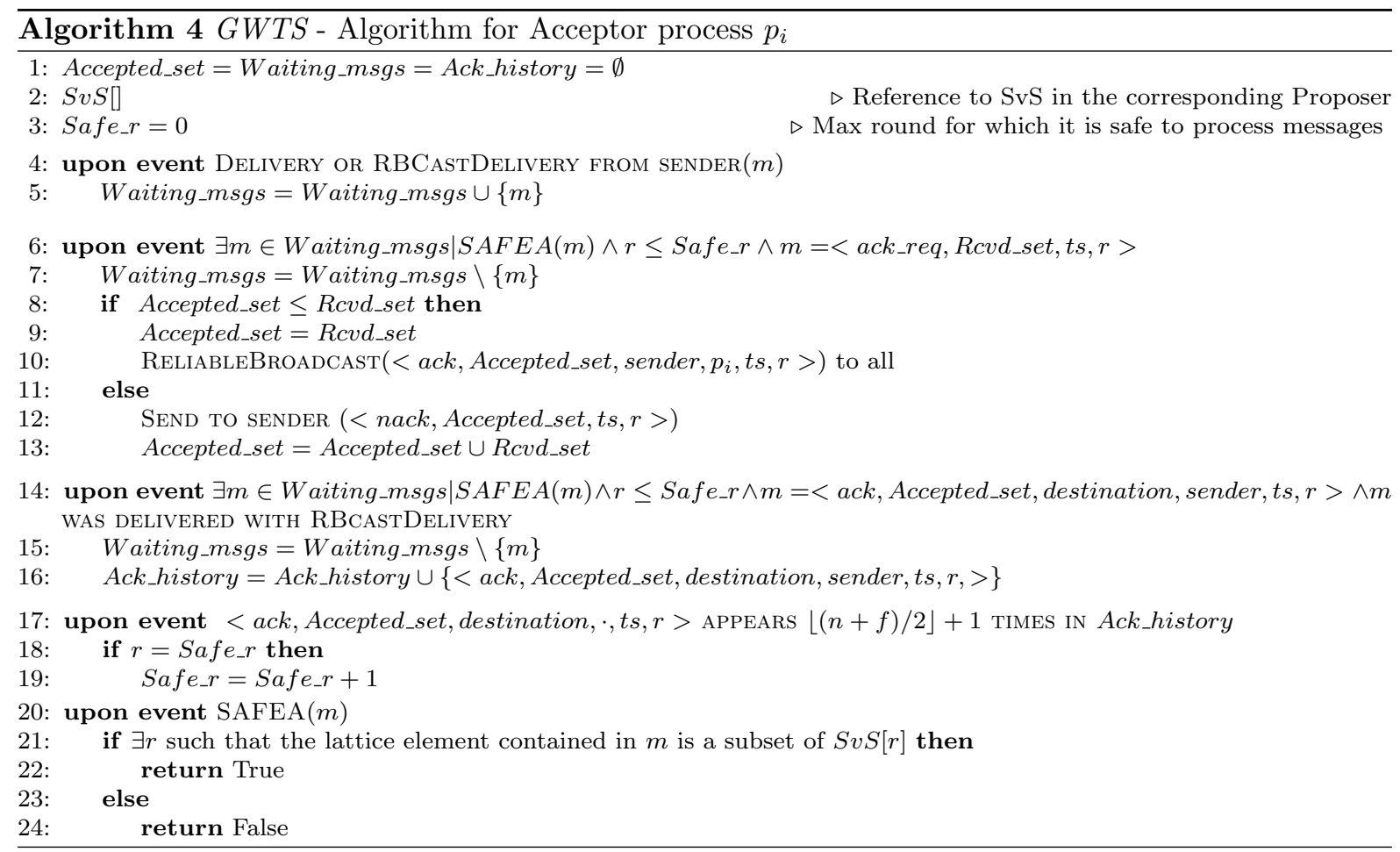

Compared to WTS, an additional challenge to be faced is to prevent adversarial processes from indefinitely postpone the decision correct processes. A uncareful design could allow byzantine proposers to continuously pretend to have decided, thus jumping to new rounds, and clogging the proposers with a continuous stream of new values. This would make acceptors to continuously nack proposals of correct processes. We solve this problem through the acceptors. Acceptors will help a new proposal to be decided in round $r \geq 1$ when, and if, in round $(r-1)$ a proposal has been accepted by at least a (Byzantine) quorum of acceptors (i.e., $s a f e \_r=r$ ). In order for this to work we make acceptors to reliably broadcast their ack messages, in this way the acceptance of proposals is made public. Any correct proposer can decide, in a round $r$, any proposal that has been correctly accepted in round $r$, even if it was not proposed by itself (provided that such decision preserves the Local Stability).

\subsection{GWTS properties}

\subsubsection{Safety properties}

The proof of the safety properties of GWTS is analogous to the proof contained in Section 5.1.1. From the properties of reliable broadcast we have the following:

Observation 3. For each correct process $p_{i}$ and each round $r$, the set $S v S[r]$ contains at most $n$ sets.

Theorem 4. Let us consider a set of processes, of size $(3 f+1)$, executing GWTS algorithm. Algorithm GWTS enforces: (1) Comparability; (2) Non-Triviality; (3) Stability.

Proof. We prove each property separately.

1. is implied by Lemma 1 and Observation 2

2. For the Non-Triviality we have to show that for each correct proposer $p_{i}$ and each $\operatorname{dec}_{k} \in D e c_{i}$ it holds $d e c_{t} \leq \bigoplus(\operatorname{Prop}[0: h] \cup B[0: b])$. First notice that $d e c_{t}$ may only contain values that are present in $\bigcup_{r^{\prime} \in[0, r]} S v S\left[r^{\prime}\right]$ with $r$ rounds in which $d e c_{k}$ happens. This derives from the fact that a correct process, at a given round, only handles messages that contain safe values. Let $W_{r}=\bigcup_{r^{\prime} \in[0, r]} S v S\left[r^{\prime}\right]$, it is immediate to see that $W_{r}$ contains at most the union of all values in the prefixes $\operatorname{Prop}_{i}[0: h]$ from some index $h$, which correspond precisely to all values proposed 
by correct processes until round $r$. It is also immediate that $W_{r}$ contains at most the union of all values that Byzantine proposers have reliably broadcast in the first $r$ disclosure phases: this is equivalent to say that it contains a prefix of all the infinite sequences of values that Byzantine cumulatively broadcast in the disclosure phases of our algorithm. From these arguments, and the check at Line 18 of Algorithm 3 the Non-Triviality follows.

3. is ensured by line 40 in proposers.

\subsubsection{Liveness}

We say that a correct process $p_{i}$ joins a round $r$ if it sends a message $<$ disclosure_phase, $\cdot r, r$. Similarly a correct process $p_{i}$ proposes a $S e t$ at round $r$ if it sends a message $\left\langle a c k_{-} r e q\right.$, Set, $\left.\cdot, r\right\rangle$.

We say that a message $m$ is safe for a process $p_{i}$ at round $r$, if $S v S[r]$ of $p_{i}$ contains of all values contained in $m$.

Lemma 5. Each message sent by a correct process at round $r$ is eventually safe, at round $r$, for any other correct process.

Proof. Same of Lemma2

Definition 3. We say that a round $r$ has a "legitimate end" if there exists a proposal that has been committed at round $r$.

(See Definition 2 of committed proposal)

Definition 4. Round $r$ is a legit round, at time $t$, if, either, $r$ is 0 or $(r-1)$ had a legitimate end before time $t$.

Definition 5. An acceptor trusts round $r$ if its Safe_r $\geq r$.

Lemma 6. If $r$ is a legit round, then eventually any correct acceptor will trust round $r$.

Proof. The proof is by induction on $r$.

- Base case: for round $r=0$ each acceptor has Safe_r initialized to 0.

- Inductive case: By inductive hypothesis we have that eventually any acceptor sets $S a f e \_r=r-1$. Moreover, we have that, by definition of legit, round $(r-1)$ had a legitimated end. This means that $2 f+1$ acceptors reliably broadcast $<$ ack, Accepted_set, destination, $\cdot, t s, r-1>$, and at least one of them is correct process $p$, thus the message $<$ ack, Accepted_set,destination, $\cdot \cdot$, ts, $r-1>$ is safe for $p$ at round $(r-1)$. Any acceptor with $S a f e_{-} r=r-1$ upon receipt of these messages will eventually process them, by Lemma 5, and it will set $S a f e \_r=r$, see procedure starting at line 17 .

Note that by Lemma 6 we have that any legit round will be eventually trusted by all acceptors. Moreover, we can show that if $r$ is a non-legit round at time $t$ than it will not be trusted, at time $t$, by any correct acceptor.

Lemma 7. If $r$ is a non-legit round, at time $t$, that is, $r \neq 0$ and $(r-1)$ has not had a legitimate end before time $t$, then any correct acceptor has Safe_r $<r$.

Proof. The proof derives immediately from the definition of legitimate end and from line 17 in the acceptor code.

Definition 6. A value $v$ has been disseminated, by time $t$, if, by time $t$, it was contained in a safe ack_req message for some round $r$ and it has been received by $\lfloor(n+f) / 2\rfloor+1$ correct acceptors that trusted round $r$.

The observation below is a strengthen version of Lemma 1 
Observation 4. If a value $v$ has been disseminated by time $t$, then any proposal committed after time $t$ will contain $v$.

Proof. The proof is immediate by observing that a disseminated value is in the Accepted_set of $\lfloor(n-$ $f) / 2\rfloor+1$ correct acceptors (either by line 9 or 13 of Algorithm 4), and by using the same argument of Lemma 1 .

Lemma 8. If round $r$ has a legitimate end and at least $(n-f)$ correct proposers joined round $r$, then eventually any correct proposer, that joined round $r$, will decide in round $r$, and join round $(r+1)$.

Proof. The proof is by contradiction. Let $p_{i}$ be a correct process that joined round $r$ but has not yet decided in round $r$. Note that $p_{i}$ has to be in state proposing: by hypothesis $(n-f)$ correct proposers joined $r$, thus the guard at line 24 has to be eventually triggered.

Since $r$ has a legitimate end then there are $\lfloor(n+f) / 2\rfloor+1$ reliable broadcast of messages < ack, Accepted_set,destination, $\cdot, t s, r>$, and at least one of them has been generated by a correct process $p$, thus it is safe for $p$ at round $r$. By Observation 4 we have that Decide_set $\subseteq$ Accepted_set, and, by Lemma 5, upon receipt of these messages $p_{i}$ decides and joins round $(r+1)$.

Lemma 9. If $r$ is a legit round, then any correct proposer eventually joins it.

Proof. The proof is by induction on round number.

- Base case: round $r=0$, by assumption it is a legit round, and by algorithm construction each correct proposer joins $r=0$.

- Inductive case: The inductive hypothesis is that $(r-1)$ is a legit round and that each correct proposer joined it. We assume that $r$ is a legit round, thus round $(r-1)$ had a legitimate end. Lemma 8 and the inductive hypothesis imply that any correct proposer joins $r$.

Lemma 10. If $r$ is a legit round, then it will eventually have a legitimate end. Moreover, each correct proposer executes line 33, while its round variable is $r$, at most $f$ times (that is it refines its proposal at most $f$ times during its participation to round $r$ ).

Proof. First observe, by Lemma 6 that each correct acceptor eventually trusts round $r$. Then observe that, until $r$ does not have a legitimate end, by Lemma 7, no correct acceptor will trust any round $r^{\prime}>r$. Thus they will not process any message coming from round $r^{\prime}$.

The above and Observation 3 bound the number of changes that correct acceptors perform in round $r$ on their Accepted_set to a finite number. Therefore, there exists a time $t$ after which each correct acceptor does not change anymore its Accepted_set.

If a correct proposer, that joined round $r$, issues an ack_req after time $t$, then, by Lemma 5 , and the above reasoning we have that such request will be committed. Once committed round $r$ has a legitimate end. Now by Lemma 9 we have that eventually any correct proposer joins round $r$. It remains to show that some correct process issues a request after time $t$. Note that, when joining a new round, each correct process proposes its value. This proposal either is committed or refined (upon execution of line 33). In case of refinement a new set is immediately proposed. This ensures that either something was committed in round $r$ before time $t$, or that something will be proposed after $t$. In both cases round $r$ will have a legitimate end. Recall that all correct proposers eventually join round $r$ (by Lemma 9p, each of them only proposes the value constituted by Batch $[r]$, by the property of the Byzantine reliable broadcast and by the safety of messages also Byzantines are constrained to propose at most $f$ different values in round $r$. This means that a decision in round $r$ can contain at most $n$ new values with respect to the decision at round $(r-1)$. Since when a correct proposer executes line 27 it passed the guard at line 24 , it is obvious that there at most $f$ values missing in its proposal. From this Lemma 10, and thus the bound on the number of executions of line 33 . follows immediately.

Lemma 11. If a correct process $p_{i}$ joins a round $r$ at time $t$, it also proposes, in round $r$, all values in Pro $_{i}$ received before time $t$. 
Proof. Observe that a correct process joins a round $r$ only if $(r-1)$ had a legitimate end. From Lemma 9 we have that all correct processes will eventually join round $r$, thus the if at line 24 will be passed. From the above, the atomicity of the local procedures, and the fact that a correct process cumulates in its Proposed_set all previous batches never removing any value (see line 20, our claim follows.

From Lemma 11 we have the following observation:

Observation 5. Given any correct process $p_{i} \in C$ and any value $v \in$ Pro $_{i}$, we have that $v$ is eventually disseminated.

Theorem 5. Let us consider a set of processes, of size $(3 f+1)$, executing GWTS algorithm. We have that any run of GWTS ensures the following liveness properties:

1. Liveness: each correct process $p_{i} \in C$ performs an infinite sequence of decisions Dec $_{i}=\left\{\right.$ dec $_{0}$, dec $_{1}$, dec $\left._{2}, \ldots\right\}$

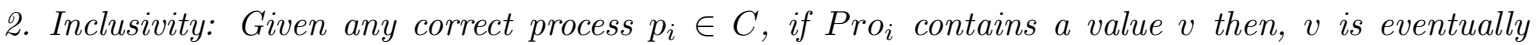
included in one decision in $D e c_{i}$;

Proof. We prove each property separately:

1. Liveness: it is enough to show that there is an infinite sequence of legit rounds. This derives from Lemma 10, combining it with Lemma 8 and a simple induction on the round number. Lemmas 8, 9 ensure that in each round of such sequence all correct proposers decide.

2. Inclusivity: it derives from Observations 5, 4 and the fact that the sequence of decisions is infinite (see above).

\subsection{Message Complexity}

GWTS executes a possibly infinite sequence of decisions. Thus, we restrict our message complexity analysis to the number of messages needed for each decision. The messages are counted per proposer, we include messages created by correct acceptors in response to proposer actions. Each proposer decides exactly once for each algorithm round. Therefore, we count messages from start to end of a generic round. A proposer has to reliably broadcast its batch (line 16 -cost $\mathcal{O}\left(n^{2}\right)$ ), it has to broadcast its proposal (line 27- cost $\mathcal{O}(n))$, then, in the worst case, it refines its proposal at most $f$ times (see Lemma 10 -line 35- cost $\mathcal{O}(n))$, however each ack from a correct acceptor has to be reliably broadcast (line 10 - cost $\left.\mathcal{O}\left(n^{2}\right)\right)$. The total cost is therefore upper-bounded by $\mathcal{O}\left(f \cdot n^{2}\right)$.

\section{Byzantine tolerant RSM}

We are interested in wait-free implementations of linearizable replicated state machines for commutative update operations in the Byzantine model.

\subsection{Specification of the Byzantine tolerant RSM}

The replicated state machine is composed of $n$ replicas, which start in the initial empty state $s_{0}$. Among them, up to $f \leq(n-1) / 3$ replicas may exhibit Byzantine failures. The RSM exposes two operations, update and read, such that the update operation with command $c m d$ modifies the current state $s$ of the RSM by applying $c m d$ to $s$ but does not return any value, while the read operation returns the current state of the RSM. The state of the RSM at time $t$ is a set of update commands applied to the initial state $s_{0}$ until time $t$. Note that being the RSM commutative the order in which updates are applied does not matter. Clients may trigger an infinite number of read and update operations. We assume that each command is unique (which can be easily done by tagging it with the identifier of the client and a sequence number). We do not make any assumptions regarding clients behavior: they can exhibit arbitrary behaviors (e.g., invoke an update operation with some arbitrary command, or modify the read and update code). We do not limit the number of Byzantine clients. Hence, to prevent Byzantine clients from jeopardizing the state of the RSM through arbitrary commands, commands are locally executed by 
clients: the RSM provides clients with a set of updates and clients locally execute them. For readibility reasons, the value returned by the execution of a set of commands is equal to the set of commands. The following properties formalise the behavior of read and update operations during any execution run by correct clients:

- Liveness Any update and read operation completes;

- Read Validity: Any value returned by a read reflects a state of the RSM;

- Read Consistency: Any two values returned by any two reads are comparable;

- Read Monotonicity: For any two reads $r_{1}$ and $r_{2}$ returning value $v_{1}$ and $v_{2}$ respectively, if $r_{1}$ completes before $r_{2}$ is triggered then $v_{1} \subseteq v_{2}$;

- Update Stability: If update $u_{1}$ completes before update $u_{2}$ is triggered then every read that returns a value that includes the command of $u_{2}$ also include the command of $u_{1}$;

- Update Visibility: If update $u$ completes before read $r$ is triggered then the value returned by $r$ includes the command of $u$.

\subsection{Implementation of the Byzantine tolerant RSM}

As previously introduced, our general idea to implement a wait-free and linearizable replicated state machine resilient to Byzantine failures in an asynchronous system is to apply Generalized Lattice Agreement on the power set of all the update commands. GWTS is executed by the replicas of the state machine (for simplicity reasons replicas play the role of both proposers and acceptors). The update and read operations are presented in Algorithms 5 and 6 respectively. The update operation consists in submitting the new command $c m d$ to generalized Lattice Agreement so that eventually the new state of each (correct) replica includes $\mathrm{cmd}$. This is achieved by triggering the execution of NEW VALUE with $\{c m d\}$ as parameter at any subset of $(f+1)$ replicas (so that at least one correct replica will execute it), see Line 3. The update operation completes when some correct replica modifies its local state with $\mathrm{cmd}$, that is, decides a decision value that includes $\mathrm{cmd}$ (Line 4). This preserves the order of non-overlapping update operations. The read operation consists in an update operation followed by a confirmation step. The update is triggered with a special value nop that locally modifies a replica's state as for an ordinary command $c m d$ but is equivalent to a nop operation when executed. When the update completes, any decision value decided by a correct replica can be returned by the read operation. Since up to $f$ Byzantine replicas may provide any value, a confirmation request for each of these $(f+1)$ decision values is sent to all replicas (Line 8). A replica acknowledges Accepted_set if Accepted_set has been accepted by $\lfloor(n+f) / 2\rfloor+1$ acceptors, which ensures that Accepted_set has effectively been decided in GWTS (Line 4 of Alg. 7). The value returned by the read operation is the result of the execution of the first decision value confirmed by $f+1$ replicas, i.e, the first decision value confirmed by at least one correct replica. This ensures that a read operation will return a value that reflects the effect of the last update operation. From an implementation point of view, the confirmation step requires to add two lines of code in Algorithm 3. Specifically, when a proposer receives a confirmation request for decision value Accepted_set, then it acknowledges the request if $<$ ack, Accepted_set, $\cdot, \cdot, t s, r>$ appears $\lfloor(n+f) / 2\rfloor+1$ times in its Ack_history set for a fixed combination of $t s$ and round $r$. See Code in Alg. 7.

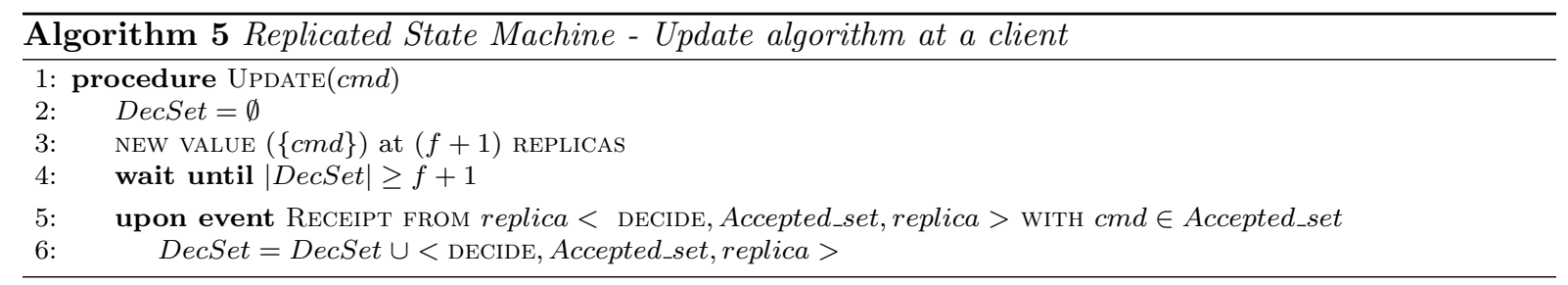



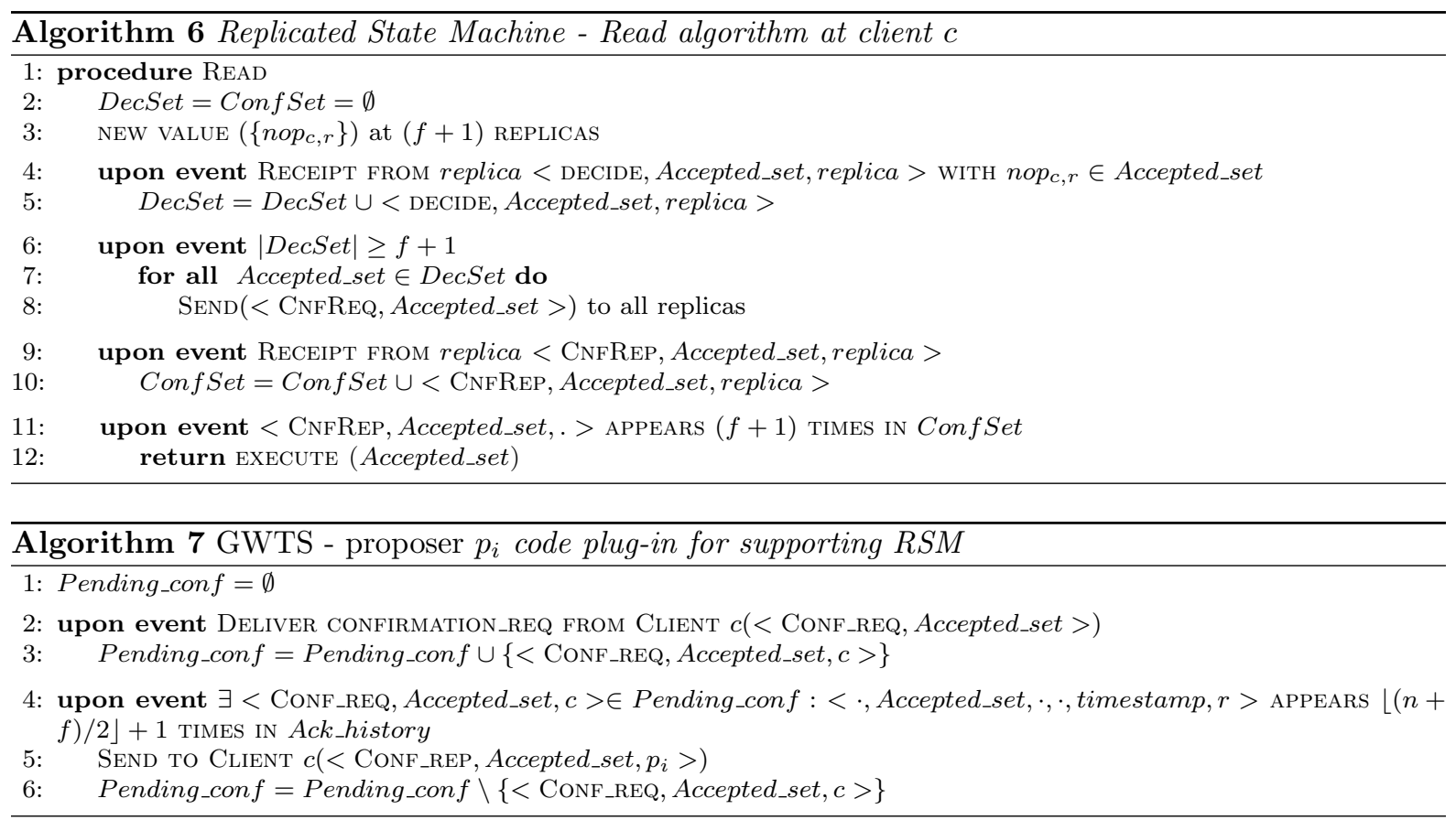

Theorem 6. Given the wait-free Byzantine generalized Lattice Agreement (GLA) algorithm whose pseu-

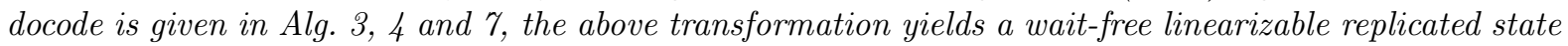
machine for commutative update operations. This transformation requires one execution of the Byzantine GLA algorithm.

Proof. The proof consists in showing that (1) liveness, (2) read validity, (3) read consistency, (4) update stability, (5) read monotonicity, and (6) update visibility properties holds. We prove each property separately.

- (1) Liveness of the update operation is straightforward from Theorem 5 . For the read operation, liveness holds from update liveness and from the fact that among the $(f+1)$ received values, at least one is the decision value of a correct replica, which by Lines 39 41 of Algorithm 3 , has been accepted and reliably broadcast to all proposers by $(2 f+1)$ acceptors, and thus reliably delivered by all proposers (by Liveness of Reliable Broadcast);

- (2) Straightforward from the fact that the value returned by a read is a decision value.

- (3) Straightforward from Theorem 4

- (4) By Observation 4 and by the fact that a read operation begins with an update operation;

- (5) By applying the same argument as for update stability, read monotonicity holds.

- (6) By applying the same argument as for update stability, update visibility holds.

Lemma 12. The above transformation is resilient to Byzantine clients.

Proof. A Byzantine client $c$ may threaten the correctness of the above transformation by uncorrectly executing the read and write operations.

- Suppose that $c$ invokes Update $(\mathrm{cmd})$. If $\mathrm{cmd}$ is not a admissible command (i.e., is not an element of the lattice) then correct replicas filter out $\mathrm{cmd}$ (line 19p, and thus the operation has no impact on the RSM state. Now, if $c$ sends $c m d$ to less than $f+1$ replicas (line 3), then it is sufficient that a single replica receives and proposes $c m d$ to GWTS for $c m d$ to be decided by all correct replicas, and thus correctly impacts the state of the RSM. Finally if $c$ invokes a sequence of updates $u_{i}, \ldots u \ell$ 
without waiting for each $u_{i}$ to complete (line 4), then these update operations will be considered as concurrent operations, which is handled by GWTS.

- Suppose that $c$ invokes Read(). The only difference with the Update() operation is the confirmation phase (lines 7. 11) which acts as a proof that the value returned by the read operation is a decided value. This phase does not impact the state of the RSM, which completes the proof of the lemma.

\section{Safety by Signature Algorithm - An algorithm with linear message complexity}

In this Section we will discuss how to decrease the message complexity using signatures. The resulting algorithm has a message complexity of $\mathcal{O}(n)$ when $f=\mathcal{O}(1)$. This Algorithm introduces a trade-off between message-complexity and message-size: it sends messages that could have size $\mathcal{O}\left(n^{2}\right)$, this is not the case with the original WTS. Pseudocode is on Algorithms 89, and 10, The algorithm is conceptually divided in three phases:

- Init: in this phase each process broadcasts a signed version of its initial proposed value. A process collects these messages until it sees $(n-f)$ of it. The purpose of this phase is to ensure that any correct process is able to create a set of values containing at least $|X \cup B|-2 f$ values from correct processes.

- Safetying: At the end of the init phase a correct process has a certain set of values. The purpose of the safetying phase is to make at least $|X \cup B|-2 f$ such values safe. In such case a value $v$ is safe if we are sure that no other process can see a different value $v^{\prime}$ that is also safe and has been sent by the same sender of $v$. Safetying is done by performing a broadcast of the $(n-f)$ signed values obtained in the init phase towards the acceptors. Each acceptor keeps a set of values that are candidate to be safe. Once an acceptor receives a set of values from a proposer, it starts processing each value contained in it. If there is a value $v$ such that it has not see another value sent by the same sender (this check is done by using signature), it adds the value $v$ to its candidate set. Otherwise, if it exists a $v^{\prime}$ from the same sender, it adds $\left(v, v^{\prime}\right)$ to a temporary set of Conflicts. The acceptor replies back to the proposer by sending a signed message that contains the set received, and the set of conflicts found. A proposer possesses a proof of safety for a value $v$ if it receives $\lfloor(n+f) / 2\rfloor+1$ messages from different acceptors in which $v$ never appears as a conflict. The intuition behind this phase is that if the same Byzantine process injects two (or more) values signed by him in the init phase, then at most one of them could manage to get a correct proof of safety.

- Proposing: This phase is identical to the proposing phase of WTS. The only difference is that a correct proposer and acceptor refuse to process any message that contains a value without an attached proof of safety.

\subsection{Algorithm properties}

We say that a value $v$ and a value $v^{\prime}$ conflict if $v \neq v^{\prime}$ and $v$.sender $=v^{\prime}$.sender. Given a set of values we say that the set is conflicting if it contains two conflicting values.

Safety. In the signature based algorithm we define a value as safe if:

Definition 7. (Safe value) $A$ value $v$ is safe if there are $\lfloor(n+f) / 2\rfloor+1$ broadcasts of messages < safe_ack, Rcvd_set,Conflicts, rts $>$ from different acceptors, such that $v$ is contained in each Rcvd_set and it is not contained in a pair inside a set Conflicts. 


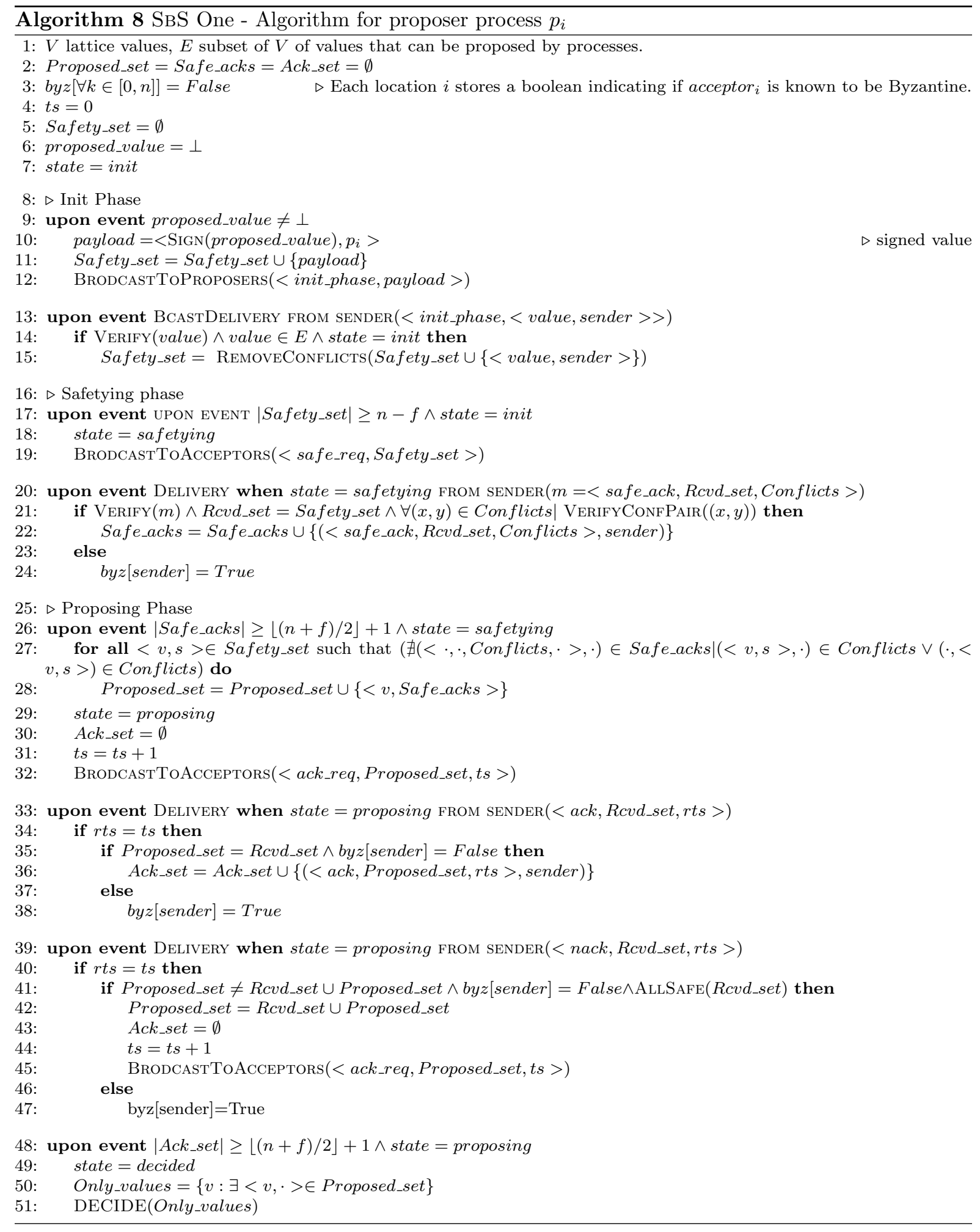



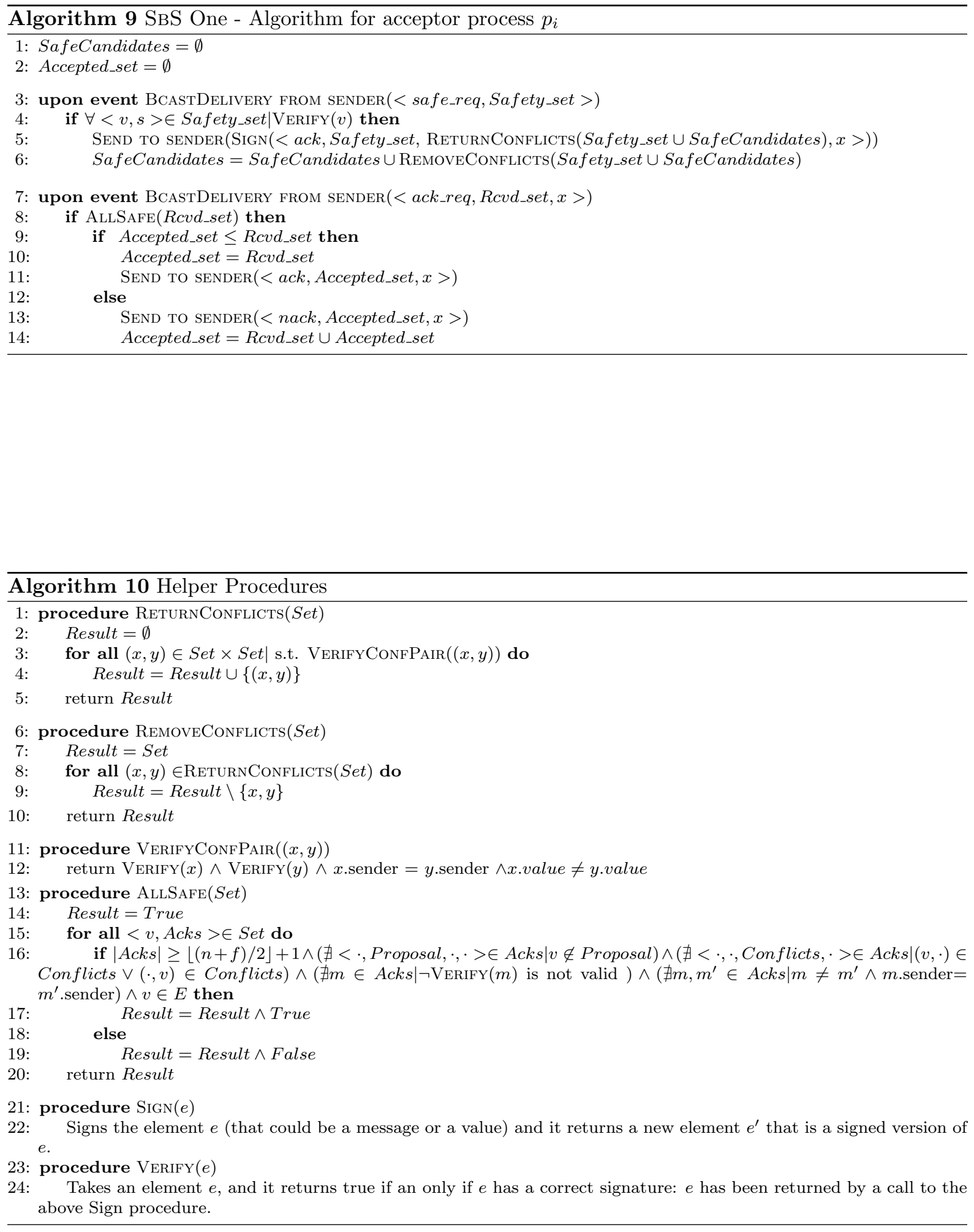
Lemma 13. Let $W_{j}$ be the set of values signed by process $p_{j} \in P$ appearing during any execution of the SbS algorithm. $W_{j}$ contains at most one safe value.

Proof. By contradiction let us assume that there are two values $x, y \in W_{j}$ both safe. Then for each value we have $\lfloor(n+f) / 2\rfloor+1$ acceptors that broadcast the safe_ack messages. Any two sets of $\lfloor(n+f) / 2\rfloor+1$ acceptors have a non empty intersection in the set of correct acceptors: there should be at least one correct acceptor $p$ that generated the message (or messages) safe_ack that made both $x$ and $y$ safe. First of all, notice that the message cannot be the same for both values (since they conflict they would be inserted in Conflicts by $p$, see line 5 of the acceptor code). For this reason we should have that $p$ has done two different broadcasts one for $x$ and one for $y$. Let us assume w.l.o.g that the first broadcast has been for $x$, note that $x$ is inserted in SafeCandidate set (line 6). This implies that once a safe_req with set containing $y$ is received by $p$ (line 3 p $p$ detects the conflict with $x$ in SafeCandidate (line 5). Therefore, $p$ cannot generate any message in which $y$ does not appear in Conflicts.

Lemma 14. Let us consider an execution of $S b S$. Let $p_{i}$ be a correct process, and let $v_{i}$ be a value that $p_{i}$ signs and inserts in its Proposed_set at line 11. If $p_{i}$ decides a set decide ${ }_{i}$, then $v_{i} \in$ decide $_{i}$.

Proof. First of all, note that $p_{i}$ is the only one that can create a valid signature on a value $v$. Therefore, if a Byzantine process creates a pair in a Conflicts set containing value $v_{i}$ for a certain message $m=<$ safe_ack,, Conflicts, $\cdot>$, then no correct process will insert $m$ in its Safe_ack set (the signature check at line 21 will not be passed). Stated otherwise, $v_{i}$ can never appear in a Conflicts set of a message $m=<$ safe_ack, $\cdot$, Conflicts, $\cdot>$ that will be processed by any correct process. This implies that, if $p_{i}$ decides, then $p_{i}$ inserted $v_{i}$ in Safe_proposal at line 28 Since, $p_{i}$ never removes any value from Proposed_set we have that an eventual decision contains $v_{i}$.

Note that the proposing phase of SbS is analogous to the one of the WTS algorithm. It is not hard to see that Lemma 1 and Observation 2 holds also for the proposing phase of SbS.

Theorem 7. Let us consider a set of processes, of size at least $(3 f+1)$, executing SbS algorithm. Algorithm SbS enforces: (1) Comparability; (2) Inclusivity; (3) Non-Triviality; (4) Stability.

Proof. We prove each property separately.

1. is implied by Lemma 1 1 . Observation 2 and the fact that a proposer refuses to process nacks containing values that are not safe (see if at line 41).

2. by Lemma 14

3. the bound on $B$ and $X$ derives from the safety of each value inserted in a decision and Lemma 13 .

4. is ensured by line 49 in the code run by proposers.

Liveness The main result of this section is a bound of $\mathcal{O}(f)$ on the number of times a proposer refines its proposal. This bound will be needed to prove that our algorithm uses a quadratic number of messages.

Lemma 15. Let us consider an execution of SbS. Any correct process eventually executes line 32, and when it does its Proposed_set contains at least $|X \cup B|-2 f$ values.

Proof. Since at most $f$ processes are faulty line 17 will be eventually executed on each proposer, and when it happens its Safety_set contains at least $|X \cup B|-f$ values. It remains to show that line 32 is eventually executed and that at most $f$ values are not inserted in Proposed_set during the loop at line 28. First of all notice that at least $(n-f)$ acceptors will reply to the safe_req sent at line 19 , this ensures that eventually the procedure at line 26 will be executed. Moreover, we have that the Sa fety_set sent at line 19 contains at most $f$ values of Byzantine processes. By the same reasoning used in the proof of Lemma 14 we have that at least $|X \cup B|-2 f$ values are from correct proposers and will be sent at line 32

Lemma 16. A correct proposer refines its proposal (executing line 45) at most $2 f$ times. 
Proof. Each time a correct proposer executes line 45, it passes the if at line 41, thus increasing its Proposed_set. However, its first proposal, in line 19 contains at least $|X \cup B|-2 f$ values. Since there are at most $|X \cup B|$ safe values (from Lemma 13 and the if at line 41 can be passed only when all values inside $R c v d \_s e t$ are safe, the claim follows.

Lemma 17. When a correct acceptor receives a message $m=<$ ack_req, Rcvd_set, $x>$ from a correct proposer it answers with an ack or a nack.

Proof. If a correct acceptor sends a message $m$ then each value in Rcvd_set is safe, thus, when $m$ is delivered by a correct acceptor, the if at line 8 will be passed and the acceptor will answer with an ack or a nack.

Mutatis mutandis, an analogous of Lemma 4 holds also for the proposing phase of SbS:

Lemma 18. If there is a time $t$ after which a correct proposer $p_{i}$ in state proposing cannot execute line 45, then $p_{i}$ eventually decides.

Proof. Let $<$ ack_req, Proposed_set,ts $>$ be the last ack request message sent by $p_{i}$. Since $p_{i}$ does not execute line 42 it means that either it does not receive any nack, or that any nack it receives does not allow him to pass the if line 41. Since $p_{i}$ is correct its message $\left\langle a c k \_r e q\right.$, Proposed_set,ts $>$ will reach each correct acceptor. By hypothesis each of them will send a ack, otherwise $p_{i}$ should be able to execute line 42 (they all handle the ack request by Lemma 17). Once $p_{i}$ receives the acks from the set of correct acceptors it will handle them, Lemma 17, and decides.

Theorem 8. Let us consider a set of processes, of size at least $(3 f+1)$, executing SbS algorithm. Every correct proposer decides in at most $5+4 f$ message delays.

Proof. The broadcast at line 12 takes at most 1 message delay. Therefore after one message delay each correct process starts its safetying phase. This phase takes two message delays: one to reach the acceptors, and one to receive the response back. Each refinement takes at most 2 message delays, the time needed to broadcast and receive a response. There are at most $2 f$ refinements, see Lemma 16 . Executed in at most $4 f$ message delays, by Lemma 18 after $5+4 f$ message delay a correct has to decide.

Message Complexity We now bound the message complexity by counting the maximum number of messages needed by a correct proposer to decide. We do it phase by phase:

- Init phase: each correct proposer executes a single broadcast. Cost $\mathcal{O}(n)$.

- Safetying phase: each correct proposer executes a single broadcast, such broadcast triggers at most one point-to-point response from each correct acceptor. Cost $\mathcal{O}(n)$.

- Proposing phase: each correct proposer executes a single broadcast for each proposal refinement and one broadcast for its initial proposal. Each of these broadcasts triggers at most one point-topoint response from each correct acceptors. Cost $\mathcal{O}(f \cdot n)$.

Summing the above costs we have a bound of $\mathcal{O}(n)$ messages.

\subsection{Generalising SbS}

Adapting the SbS algorithm to its generalised version, while keeping the message complexity improvement, needs a special attention to substitute the reliable broadcast used to acknowledge in the GWT (line 10 of Algorithm 4). We would like to replace such broadcast with a single point-to-point message.

Conceptually, that reliable broadcast has two functions: (1) it implicitly disseminates to all proposers the fact that someone is able to decide in a certain round (sharing also a possible decision value), (2) it forces a Byzantine process to broadcast the same ack message to all proposers, enforcing the "publicity" of such action.

These two functions can be replaced by two modifications. The second function could be replaced by forcing an acceptor to sign its, now poin-to-point, ack. Intuitively this allows a proposer to prove others that it received a certain ack for its proposal request. The first function is replaced by letting any correct proposer broadcast a special decided message before deciding, such message has attached 
all the acks used to decide. This would allow proposers and acceptors that receive a decided message to know that the sender of such message was allowed to decide by the algorithm rules (recall that acks are now signed). Similarly to what happen in GWTS, a round $r$ ends when someone broadcasts a wellformed decided message for that round. Where with well-formed we mean that such decided message has attached $\lfloor(n+f) / 2\rfloor+1$ signed acks. Therefore, a correct acceptor will trust a round $r$, only if it trusted round $(r-1)$ and it knows that rounds $(r-1)$ terminated (this knowledge derives from seeing a decided message for round $(r-1))$. Finally, to avoid the reliable broadcast of decided messages, a correct acceptor piggybacks a decided message, that it has seen for a round $r$, in the replies to ack_requests for round $r$.

Message Complexity The message complexity follows the same lines of the analysis in Section 6.4. However, since we are not using the Byzantine reliable broadcast, messages per decision on each proposer are at $\operatorname{most} \mathcal{O}(f \cdot n)$.

\section{Conclusions}

We investigated Byzantine Lattice Agreement and we used it to build a byzantine tolerant RSM with commutative updated. There are several directions meriting investigation. Our main interest is to understand whether a message delay of $\mathcal{O}(f)$ is necessary or not. In the crash-stop model a recent paper 15] has shown that $\mathcal{O}(\log f)$ delays are sufficient. Therefore, a first step would be to investigate if the technique used in [15] could be "Byzantined" while preserving the desirable delay. A final target is to understand the necessary number of message delays. This would be extremely interesting, even in the crash-stop model such knowledge is still missing.

\section{References}

[1] M. J. Fischer, N. A. Lynch, and M. S. Paterson, "Impossibility of distributed consensus with one faulty process," Journal of the ACM, vol. 32, no. 2, 1985.

[2] J. Faleiro, S. Rajamani, K. Rajan, G. Ramalingam, and K. Vaswani, "Generalized lattice agreement," in Proceedings of the ACM Principles of Distributed Computing (PODC), 2012.

[3] N. M. Preguiça, C. Baquero, and M. Shapiro, "Conflict-free replicated data types crdts," in Encyclopedia of Big Data Technologies, 2019.

[4] H. Attiya, M. Herlihy, and O. Rachman, "Atomic snaphots using lattice agreement," Distributed Computing, vol. 8, pp. 121-132, 1995.

[5] X. Zheng, C. Hu, and V. K. Garg, "Lattice agreement in message passing systems," in Proceedings of the International Symposium on Distributed Computing (DISC), 2018.

[6] J. Skrzypczak, F. Schintke, and T. Schuütt, "Brief announcement: Linearizable state machine replication of state-based crdts without logs," in Proceedings of the ACM Principles of Distributed Computing (PODC), 2019.

[7] T. Nowak and J. Rybicki, "Byzantine approximate agreement on graphs," in Proceedings of the International Symposium on Distributed Computing (DISC), 2019.

[8] R. Kotla, L. Alvisi, M. Dahlin, A. Clement, and E. Wong, "Zyzzyva: speculative byzantine fault tolerance," in ACM SIGOPS Operating Systems Review, vol. 41, no. 6. ACM, 2007, pp. 45-58.

[9] A. J. H., "Composite registers," Distributed Computing, vol. 6, no. 3, pp. 141-154, 1993.

[10] Y. Afek, H. Attiya, D. Dolev, E. Gafni, M. Merritt, and N. Shavit, "Atomic snapshots of shared memory," Journal of the ACM, vol. 40, no. 3, pp. 973-890, 1993.

[11] J. B. Nation, "Notes on lattice theory," http://math.hawaii.edu/ jb/math618/NationLatticeTheory.pdf. 
[12] G. Bracha, "Asynchronous byzantine agreement protocols," Information and Computation, vol. 75, no. $2,1987$.

[13] T. K. Srikanth and S. Toueg, "Simulating authenticated broadcasts to derive simple fault-tolerant algorithms," Distributed Computing, vol. 2, no. 2, 1987.

[14] H. Mendes, M. Herlihy, N. H. Vaidya, and V. K. Garg, "Multidimensional agreement in byzantine systems," Distributed Computing, vol. 28, no. 6, pp. 423-441, 2015.

[15] X. Zheng, V. K. Garg, and J. Kaippallimalil, "Linearizable replicated state machines with lattice agreement," https://arxiv.org/abs/1810.05871, 2018, arXiv:1810.05871. 